\title{
The value of online information for demand response in Walrasian electricity markets
}

\author{
Felix Claessen*, Bart Liefers*, Michael Kaisers* and Han La Poutré* ${ }^{*}$ \\ * Centrum Wiskunde \& Informatica (CWI), Amsterdam, The Netherlands \\ $\dagger$ Technical University Delft, The Netherlands
}

\begin{abstract}
Smart energy systems integrate renewables and demand response. Most European electricity markets coordinate the resulting time-varying flexibility in demand and supply by organising day-ahead trade with Walrasian mechanisms, using simultaneous call auctions and sealed bids. These mechanisms give bidders no information on each other's values and flexibilities until after clearing. In this paper we simulate two alternative day-ahead market mechanisms which share information, such that bidders obtain a better position before entering the intraday market. One mechanism uses an ascending shared market price signal rather than sealed bids. The other auctions off future timeslots consecutively rather than simultaneously. We perform a case study on 400 households with electric vehicles, either with or without volatile wind generation. Results show that a pricetaking flexible consumer can obtain higher utility in the market with simultaneous ascending-price auctions, because online price information reduces uncertainty over available energy and prices.
\end{abstract}

Index Terms-decentralised control, energy management, power system economics, smart grids

\section{INTRODUCTION}

The changing energy system in which many distributed energy resources are present needs new and smart organisational mechanisms. Markets are especially useful for managing the fast decisions that have to be made throughout the system. Different market mechanisms have different characteristics in terms of stability, scalability and information sharing, and researchers are studying them to help policy makers, companies and system operators in shaping the emerging smart grid [1].

In this paper, we focus particularly on the characteristic of information sharing. This characteristic is receiving increased attention since the EU has passed regulation on wholesale energy market integrity and transparency (REMIT), requiring traders to share information such as their installed capacity [2]. Several auction rules strongly influence what information is available, such as the order in which bids are matched (e.g. call auctions or continuous auctions), the order in which delivery timeslots are auctioned off (e.g. consecutively or simultaneously) and the format in which bids are accepted (e.g. open or sealed). To help create a concise classification, we use the term Walrasian auction to describe a two-sided uniformprice multi-unit auction. This means both buyers and sellers place bids and the market is cleared at an equilibrium price at which demand and supply match. European day-ahead markets

Parts of this research have been funded by EIT Digital (formerly EIT ICT Labs), and excerpts have been contained in deliverable reports. can then be described as Simultaneous Sealed-bid Walrasian (SSW) call auctions (see Section II-A). Here we investigate the effects of timeslot order (consecutive vs. simultaneously) and bid format (sealed vs. ascending prices) in call auctions used for day-ahead trading. Of the four possible combinations (Table I), we present results for the Consecutive Sealedbid Walrasian (CSW) market - an alternative based on the standard method of auctioning off individual days in current electricity markets - and propose the Simultaneous Ascendingprice Walrasian (SAW) market - a refined mechanism with promising simulation performance.

The SAW market shows similarities to a market mechanism described by Gerding et al. [3], which uses simultaneous Vickrey auctions, and a simultaneous ascending-price mechanism described by Cramton et al. [4] that unlike in our mechanism, closes auctions for all timeslots at the same time. Gerding et al. chose the sealed-bid format for its low communication overhead. In contrast, we as well as Cramton et al. have chosen an iterative ascending-price format for its ability to provide online information during operation of the market mechanism. This enables bidders to effectively share information on their desired planning. The closest related mechanism is the Japanese auction for multiple goods [5] - a one-sided ascending price auction which the SAW mechanism extends to bilateral participation of both buyers and sellers, publishing also quantity imbalances at the current price. This paper provides empirical evidence that this online information improves price forecasts, and thereby the planning and utility obtained by price-taking flexible consumers.

We explain the CSW and SAW market mechanisms in detail and present our experimental method for comparing them in Section II. Results of our simulations and a discussion follow

Table I: Four categories of (W)alrasian call auctions with different timeslot ordering and bid formats, and their effects on available information. Results are presented for the CSW and SAW markets.

\begin{tabular}{lll}
\hline & (C)onsecutive slots & (S)imultaneous slots \\
\hline (S)ealed bids & CSW & SSW \\
& $\bullet$ slots traded one by one & $\bullet$ no information \\
& $\bullet$ information available & exchange about \\
& about previous slots & any slots \\
(A)scending & CAW & SAW \\
prices & $\bullet$ information available & $\bullet$ iterative scheduling \\
& about current and & $\bullet$ information available \\
& previous slots & about all slots \\
\hline
\end{tabular}


in Section III and IV, respectively.

\section{MethoD}

The performance of an electricity market for smart energy systems depends on the interplay between the market's mechanism, the bid strategies of participants, and on specific conditions such as forecast accuracy. Our experiment is designed to validate and compare the CSW and SAW markets in two scenarios, within each of which we evaluate two bidding strategies. The mechanisms, scenarios and bid strategies are described in Section II-A, II-B and II-C, respectively.

Supply and demand need to be in approximate balance at all times in the electricity grid. Ahead trading is the widely adopted coordination mechanism, and is typically facilitated by over-the-counter, day-ahead and intraday markets over predefined timeslots. Over-the-counter trades are bilateral agreements between suppliers and consumers, and the other markets are mediated by an energy exchange, like the APX or EEX power spot markets. The exchange markets may close one day ahead of delivery (day-ahead) or shortly before delivery (intraday). Here, we investigate two designs for a day-ahead market in which both buyers and sellers trade energy for multiple future timeslots of 15 minutes in the next day. We classify the market's mechanism by stating the type of auction used to trade energy for each timeslot, and the order in which the auctions are held. To make our classification concise, we use the term Walrasian market to describe a two-sided uniformprice multi-unit auction. In such an auction, a number of units of energy is traded for a given timeslot by setting a uniform market price at which demand matches supply.

The market designs are compared in simulation using two stylised cases with data generated by Flex Street [6], a model for creating demand and supply patterns of residential microgrids. The case studies demonstrate the strong and weak points of the two market designs.

\section{A. Two market mechanisms}

The Walrasian market mechanism is the standard model adopted by the majority of European electricity spot markets (e.g. APX, EEX) [7], [8]. Energy is traded each day for discrete timeslots $j \in\left\{1, \ldots, j_{\max }\right\}$. The market is a futures market for 24 hours ahead (starting at midnight) with timeslots of 15 minutes, and uses either CSW or SAW auctions for all timeslots. An overview is given in Table I.

1) CSW market: The Consecutive Sealed-bid Walrasian (CSW) market is similar to the day-ahead market, in terms of how individual days are auctioned off one after the other. With our mechanism, however, individual timeslots are auctioned off one after the other, such that information is available about all previous slots (and not just about slots in previous days). This is unlike usual day-ahead markets, which typically close all timeslots of the next day at the same time. According to our classification, the CSW market mechanism consists of sequential identical auctions, which are sealed-bid Walrasian auctions. Formally, the auctions are carried out consecutively

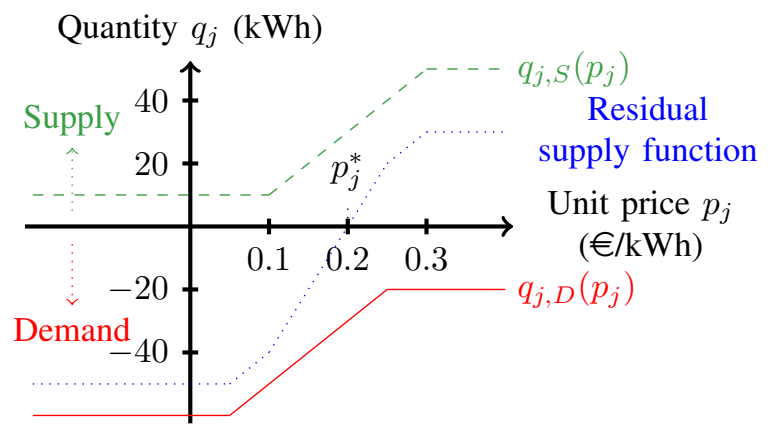

Figure 1: In the CSW market, the clearing price $p_{j}^{*}$ for a timeslot $j$ is determined by matching the bids of participating agents. Both supply bids $q_{j, S}(p)$ and demand bids $q_{j, D}(p)$ are monotonously increasing functions mapping a unit price to a quantity of energy. The residual supply function describes the excess energy (in sum) at various prices, and crosses zero at $p_{j}^{*}$.

for timeslots $j \in\{1, \ldots, 96\}$. The auction for slot $j+1$ only starts after the one for slot $j$ has closed.

For each timeslot, one auction is carried out. Agents bid in the auction by submitting a bid function, mapping a unit price $p_{j}$ (e.g. in $€ / \mathrm{kWh}$ ) to a quantity of energy $q_{j}$ (e.g. in $\mathrm{kWh}$ ) on a given timeslot $j$. Here $q_{j}>0$ indicates supply, and $q_{j}<0$ indicates demand of energy by the agent, as indicated in Figure 1.

The market sums all bid functions and calculates the unit price $p_{j}^{*}$ at which the market clears (i.e. where supply matches demand and the sum of all bid functions is zero). Given the agent models in Section II-B, the market will always clear. The agents will then be committed to the quantity that they bid at $p_{j}^{*}$.

2) SAW market: The Simultaneous Ascending-price Walrasian (SAW) market has, to our best knowledge, not been used before in electricity markets. The SAW market mechanism consists of 96 simultaneous identical auctions, which are ascending-price Walrasian auctions carried out for each timeslot $j \in\{1, \ldots, 96\}$. Each auction consists of an iterative process in which the unit price for a timeslot $j$ ascends until a clearing price is reached, as indicated in Figure 2. When a clearing price is reached, the open timeslot (with trade in progress) becomes a closed slot (with trade completed). The auctions are synchronised, such that, for a given iteration, all open timeslots share the same unit price. Note that closed slots may have different unit prices. We refer to each iteration $i$ as one market round, for which $p_{i}$ denotes the current unit price vector for all slots, i.e. $p_{i, j}$ is the current price for slot $j$. During a market round, each agent can post a bid schedule: a quantity for each timeslot, which the agent is willing to trade at the current unit price.

For the first round $(i=0)$, the unit price vector $p_{0}$ (in $€ / \mathrm{kWh})$ is set to the same starting value for each timeslot. Every agent $a$ posts a bid schedule consisting of a quantity $q_{0, j}^{a}$ for each timeslot $j$ that it is willing to trade at unit price $p_{0}$. After each round, for each open slot, $p_{i+1, j}=p_{i, j}+\Delta p$, where $\Delta p$ is a positive scalar increment of the price. 


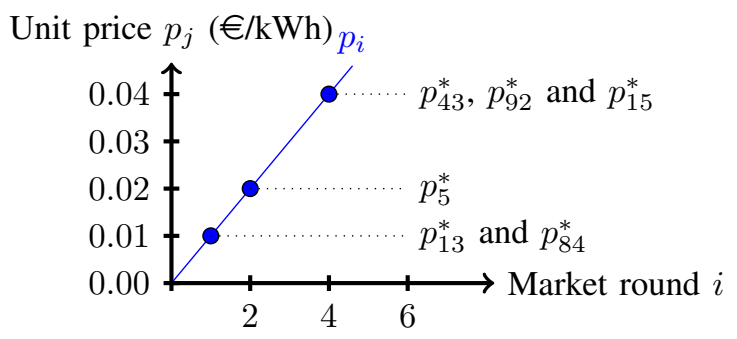

Figure 2: In the SAW market, clearing prices $p_{j}^{*}$ for timeslots $j$ are determined by incrementing the unit price $p_{i}$ for each open timeslot; when supply exceeds demand for a given timeslot, the slot is closed for trading. In this example, timeslots 13 and 84 closed first at a clearing price of $1 \mathrm{cent} / \mathrm{kWh}$.

After collecting all bid schedules for a given round $i$, the market determines the net imbalance $N_{i, j}$ for each timeslot $j$ :

$$
N_{i, j}=\sum_{a} q_{i, j}^{a}
$$

Here $N_{i, j}<0$ indicates a shortage of energy, and $N_{i, j}>$ 0 indicates an excess. For each timeslot with excess energy, demand bids become final commitments to consume at the unit price $p_{i, j}$, after which the slot is closed for trading. On each closed slot, the total supply required to cover the total demand is divided over the supply bids, according to the ratios of their bids. ${ }^{1}$

The results of each round (final commitments for closed timeslots and net imbalances for open timeslots) are communicated back to the agents, and agents are requested to update their bids. The price is increased with every new round, and agents can bid on open timeslots until all timeslots are closed.

\section{B. Scenarios $w /$ and w/o volatile supply}

We compare the market mechanisms by simulating microgrids in two stylised scenarios: $w /$ (with wind) and $w / o$ (without wind). For both scenarios, data is generated by the Flex Street model [6], and its method describing case study simulations is followed accordingly. The microgrid consists of 400 households and 400 electric vehicles (EVs). In the w/ scenario, the microgrid includes an additional wind turbine. Scenario w/o is without wind turbine. The microgrid has a single connection to the main grid, and, at any given moment, is a net consumer. The main grid is modelled as a large conventional generator, with a flexible supply large enough to cover the microgrid's peak demand.

All loads and generators are represented as individual agents, trading electricity through a local electricity market which sets electricity prices for the microgrid. The local market is of sufficient size to guarantee a low market impact for individual households and EVs. Furthermore, the aggregate consumption of 400 households gives a smooth and predictable pattern, even though individual residential patterns

\footnotetext{
${ }^{1}$ Note that in this case suppliers may be allocated less than what they bid for. Assuming suppliers have non-decreasing marginal costs and fine-grained control over their output level (e.g. conventional generation), this will be in their advantage, meaning that their utility is higher than the utility they would obtain if they were allocated their complete bid.
}

are stochastic. The two scenarios lead to a difference in market price volatility due to the stochastic generation of the wind turbine. Consequently, market prices in the w/ scenario are more difficult to forecast. Faced with this market setting, each household owner needs to procure energy according to its nonflexible (residential) and flexible (EV) demand. Likewise, each generator needs to sell energy according to its non-flexible (wind) or flexible (conventional) supply.

\section{Bid strategies}

Each household owner has the ability to adjust its EV's consumption schedule for the next day in order to maximise its utility. For this purpose we define an autonomous agent for each household's EV. It can decide its own schedule $\vec{q}$, which is a set of quantities $q_{j}$ for each timeslot $j$ of the next day. In this paper we focus on an individual household and work out a bid strategy that maximises its utility. The other agents are considered non-flexible for control purposes in the experiments. Strategies for these agents are given at the end of this section (subsection 6).

We first define the agent's constraints and valuation model for flexible EVs, from which we determine an optimal schedule for a given set of forecasted prices $\overrightarrow{\hat{p}}$. This information then allows us to construct either a bid schedule (for the SAW market) or a bid function (for the CSW market).

1) Agent constraints: The charging schedule of a vehicle's battery is controlled by an intelligent agent $a$. We assume that the battery is connected to the grid in an empty state at $5 \mathrm{pm}$ and disconnected at 9am the next morning. The agent is not allowed to deliver power back to the grid. The rate of charge is constrained between 0 and $3.7 \mathrm{~kW}$, due to the residentially typical 16 A fuse with a line voltage of $230 \mathrm{~V}$ in Europe. The maximum amount of charge in the battery is $10 \mathrm{kWh}$, which yields constraints for trading an energy quantity $q_{j}$ (in $\mathrm{kWh}$ ) for a given timeslot $j$ (of 15 minutes) formalised in (4).

2) Valuation model: To describe what a given battery level is worth to the vehicle's owner, we use a quadratic valuation function (with diminishing returns). This models that every additional unit of consumed energy has a lower contribution to the total valuation than the previous unit, i.e. non-increasing marginal value [9]. The valuation function $V$ is given by:

$$
V=B \cdot Q-A \cdot Q^{2} \quad, \quad Q=-\sum_{j} q_{j} \geq 0
$$

where each $q_{j}$ is a traded quantity (always negative for consumption) such that $Q$ is the total electricity demand in $\mathrm{kWh}$ over all timeslots $j$. $A$ and $B$ are the agent's constants for the valuation function (identical for all $\mathrm{EV}$ owners), shown in Figure 3 with the chosen experimental parameters $B=40$ cents $/ \mathrm{kWh}$ and $A=2$ cents $/(\mathrm{kWh})^{2}$.

3) Optimal schedule under forecasted prices: Given a set of forecasted unit prices $\overrightarrow{\hat{p}}$, the agent can determine its optimal schedule $\vec{q}$ by maximising its utility $\mathrm{U}$, defined as:

$$
U=V-T C \quad, \quad T C=-\overrightarrow{\hat{p}} \cdot \vec{q}
$$




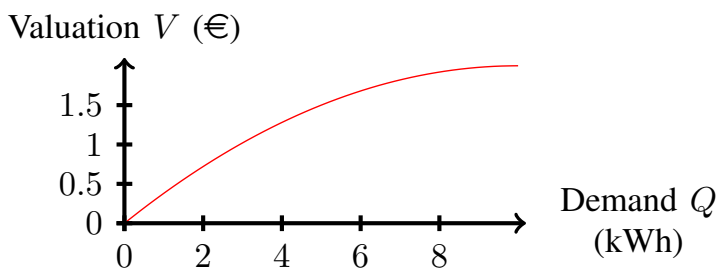

Figure 3: Valuation function for the vehicle's electricity demand.

where $T C$ are the total costs (for any timeslot $j$, costs are positive for negative $q_{j}$ ). We assume that $p_{j}$ does not depend on $q_{j}$ (negligible market impact), and write our optimisation problem as:

$$
\begin{array}{ll}
\text { maximise: } & \sum_{j=1}^{96}\left(\left(\hat{p}_{j}-B\right) \cdot q_{j}\right)-A \cdot\left(\sum_{j=1}^{96} q_{j}\right)^{2} \\
\text { subject to: } & -0.92 \leq q_{j} \leq 0 \quad, \quad-10 \quad \leq \sum_{k=1}^{j} q_{k} \leq 0
\end{array}
$$

This quadratic programme can be solved by numerical optimisation, e.g. gradient descent, which finds the optimal quantity $q_{j}$ for each timeslot given the price prediction $\overrightarrow{\hat{p}}^{2}$ This price prediction consists of:

- known market clearing prices $p_{j}^{*}$ and agent commitments $q_{j}^{*}$, for all closed timeslots

- forecasted prices $\hat{p}_{j}$ for all open timeslots. ${ }^{3}$

4) Optimal bid under forecasted prices: To bid on one of the markets we construct either a bid schedule (for the SAW market) or a bid function (for the CSW market).

In a SAW market, we let the agent post a schedule that optimises (4) at each round, given the forecasted prices of that round. We present two bid strategies: In the online SAW bid strategy, the schedule is updated after each round by reoptimising for the online price forecasts (i.e. forecasts employing the online information that becomes available during operation of the market mechanism). In the offline SAW bid strategy, the agent doesn't use online information other than 1) commitments for closed timeslots, 2) final prices for closed timeslots, 3) the current ascending price for all open timeslots. How online and offline price forecasts are constructed is explained in Section II-C5.

In a CSW market, one timeslot is handled per round, and bids should specify a quantity for that timeslot for each possible price (a bid function). The agent posts a function that optimises (4) for timeslot $i$, given the forecasted prices $\hat{p}_{j}$ for all timeslots $j \neq i$. Assuming independent prices:

$$
\frac{\partial p_{j}}{\partial p_{i}}=0 \quad \forall \quad j \neq i
$$

\footnotetext{
${ }^{2}$ If uncertainty or error information is available, the optimal strategy may deviate by taking error distribution into account.

${ }^{3}$ Except for slots where $\hat{p}_{j}<p_{i, j}$, for which it is known that the final clearing price must be at least the current round price $p_{i, j}$. This only applies to the SAW market.
}

the Lagrangian method of constrained optimisation yields an optimal quantity $q_{i}$ as a function of the actual market price $p_{i}$, according to:

$$
\begin{aligned}
q_{i}\left(p_{i}\right) & = \begin{cases}q_{i}^{\text {min }} & \text { if } p_{i}>p_{i}^{\text {max }} \\
q_{i}^{\text {max }} & \text { if } p_{i}<p_{i}^{\text {min }} \\
\frac{B-p_{i}}{2 A}-\sum_{j \neq i} \hat{q}_{j} & \text { otherwise }\end{cases} \\
p_{i}^{\text {min }} & =B-2 A\left(q_{i}^{\text {max }}+\sum_{j \neq i} \hat{q}_{j}\right) \\
p_{i}^{\text {max }} & =B-2 A\left(q_{i}^{\text {min }}+\sum_{j \neq i} \hat{q}_{j}\right)
\end{aligned}
$$

where $\hat{q}_{j}$ is the forecasted optimal quantity for timeslot $j$ given in (5), and $q_{i}^{\min }$ and $q_{i}^{\max }$ are deduced from the constraints in (4).

5) Learning to forecast prices: The forecasted prices, required by the agent to determine its optimal bid for each timeslot, can be constructed by learning from historical datapossibly in combination with external data sources such as weather forecasts - or from market quotes fed back during the market process-giving information on the valuation of other bidders. We developed forecasting methods that use a combination of historical prices (without external information) and market quotes if available. In a CSW market, no quotes are available and only historical prices are used to generate a forecast for open timeslots. We refer to this as an offline forecast. In a SAW market, initial quotes for all open timeslots become available after the first round. If these quotes are used, we refer to the resulting forecast as an online forecast.

The offline price forecast is computed from a weighted sum of the historically observed prices for the same timeslot of the last $n$ days, in which the weights decrease exponentially:

$$
w_{k}=\frac{(1-r) r^{k-1}}{1-r^{n}} \quad, \quad k=1, \ldots, n
$$

where $w_{k}$ is the weight for the $k$-th last available price, and $r$ defines the ratio between consecutive weights $\left(r=w_{k+1} / w_{k}\right)$. In the experiment $n=10$ and $r=0.5$.

Our method of constructing the online price forecast is to search historical data on market quotes. The historical data of the SAW market consists of quotes $N_{i, j, k}$, i.e. the net imbalance for each round $i$ for a given timeslot $j$ in a previous day $k$. To construct the forecast, we compare the quote sequence $N_{j, 0}$ for the current market day $(k=0)$ to the quote sequence $N_{j, k}$ for previous days. A nearest neighbour classification finds the closest matching historical quote sequence. The final price in that sequence is taken as the forecasted price for the given timeslot. Only if this gives a lower price than the price of the current market round, the round price is used instead. In the SAW market, the forecast can be updated during the iterative process. With each round, the current quote sequence becomes longer and we expect that our search through the historical data yields a more accurate forecast. 
6) Other agents: The models used for the other agents in the simulations, such as generators and inflexible consumers, do not employ learning. The flexible generator always posts the same bid function representing its marginal costs $M C$ given by:

$$
M C=A \cdot q_{j}+B
$$

where $q_{j}$ is the supplied amount of electricity in kWh during future timeslot $j$, and $A$ and $B$ are the generator's constants for marginal costs. In all cases, $B=1$ cent $/ \mathrm{kWh}$ and $A$ is set to give a daily average unit price of 20 cents $/ \mathrm{kWh}$. This bid function models that every additional unit will cost more to produce than previous units (cheapest production first).

Both the production schedule of the wind turbine and the consumption schedules of households and non-flexible EVs are price inelastic. Agents representing these entities bid fixed demands for each timeslot, regardless of price, which models the current non-adaptability of consumers and wind farms. ${ }^{4}$ According to the Flex Street model [6], the residential consumption schedules were generated by imposing a stochastic deviation on the time of use-events. The non-flexible EV schedules were generated by stochastic variation in the arrival time of the vehicles.

\section{Evaluation}

We evaluate the performance of both bidding strategies in both scenarios for each day, by comparing its obtained utility to the utility it would have obtained if it had had a perfectly accurate price forecast, i.e. when:

$$
\hat{p}_{j}=p_{j}^{*}
$$

where $\hat{p}_{j}$ is the forecasted price for timeslot $j$ used in the constrained optimisation, and $p_{j}^{*}$ is the actual clearing price. In our case, the clearing price $p_{j}^{*}$ is effectively determined by the marginal cost function $M C(q)$ of the generator defined in (8), and the demand bids $q_{j}^{a}$ and $q_{j}^{r e s t}$ of the intelligent agent and of all other agents, respectively, such that:

$$
p_{j}^{*}=M C\left(-q_{j}^{r e s t}-q_{j}^{a}\right)
$$

This actual clearing price corresponds to a perfectly accurate price forecast. Following the same procedure as in Section II-C3, we solve (4) with a perfect price forecast, which gives us the optimal utility that can be obtained by the agent. In our results, performance indicates the ratio of the agent's obtained utility with respect to the optimal utility.

\section{EXPERIMENTS AND RESULTS}

\section{A. Simulation parameters}

The simulation comprises 100 days in both cases (w/ and w/o), for which demand and supply patterns were generated stochastically. During this time, the intelligent agent controlling the flexible EV collects price observations and thus learns to forecast prices more accurately. Each day, a market is organised for the next day. For the SAW market, a price increment of 0.1 cents per round is used.

\footnotetext{
${ }^{4}$ Curtailment is politically undesired, and e.g. in Germany limited by law.
}

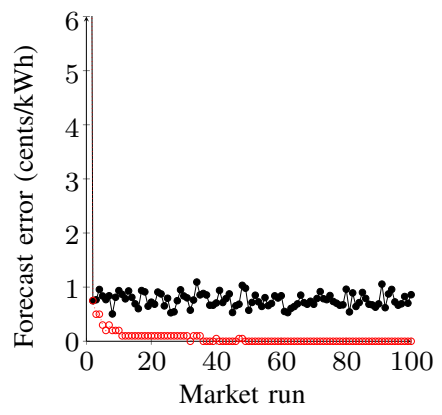

(a) Case w/o

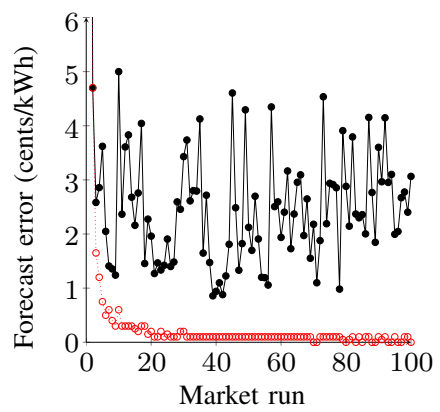

(b) Case w/
Figure 4: Price forecast error, representing the median of the absolute difference between the actual clearing price and the price forecast for each timeslot. The red circles (dotted line) represent the error of the online forecast; the black disks (solid line) represent the error of the offline forecast.

\section{B. Results}

Figure 4 shows the accuracy of the two implemented price forecast methods. Historical data becomes available after the first day, after which the error of offline forecasts shows no downward trend. In all cases, the accuracy of online price forecasts increases further over time. The first 20 days served as a start-up phase to get reliable price forecasts. The analysis in this section was performed on the remaining 80 days.

Figure 5 shows the obtained utility and performance for all simulations. The spread in the obtained utility is caused by daily differences in the demand schedules of loads and, in the w/ case, the supply schedule of the wind turbine, where a higher utility can be obtained during windy days. The agent's performance in both cases is highest in a SAW market when online forecasts are used; price volatility then has hardly any influence. When the agent's bid strategy uses only offline information, performance is lower in the volatile case for both

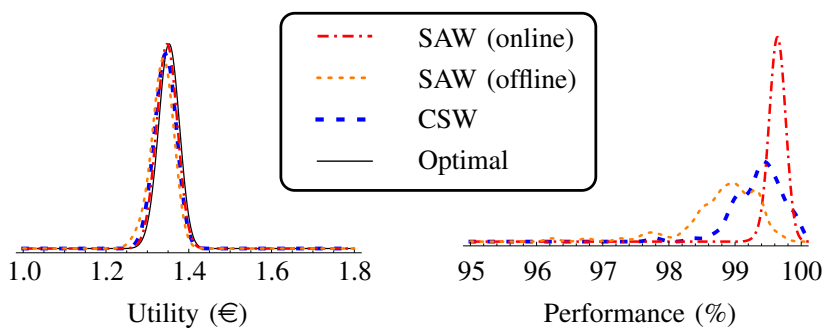

(a) Case w/o
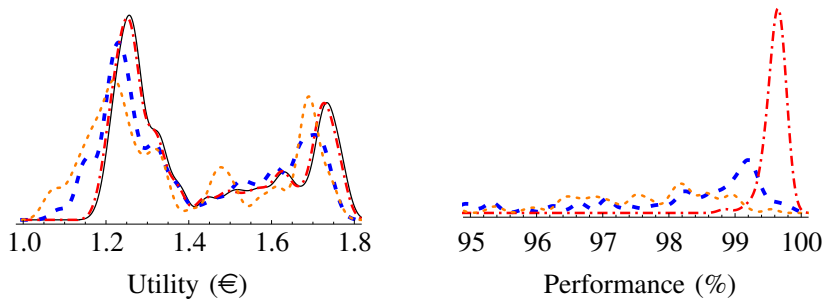

(b) Case w/

Figure 5: Distribution of the utilities and performances over all simulated days. The graphs can be interpreted as the probability of occurrence of a certain utility or performance (the total area under each curve is equal to one). The performance of the optimal allocation equals $100 \%$ by definition. 
mechanisms.

Lower utilities occur especially when offline information is used that wrongly predicts the time at which prices are lowest (Figure 6). In the w/o case (left-hand-side figures), offline forecasts accurately predict lowest prices from about $5 \mathrm{am}$ to 8am, and the agent obtains a utility that is close to optimal. In the w/ case (right-hand-side figures), due to unexpected supply from the wind turbine, the lowest price actually occurred between $4 \mathrm{am}$ and 6.30am. On the CSW market, the agent's bid function allowed it to adapt somewhat to this unexpected early opportunity, but on the SAW market without the online update, the cheapest timeslots closed without any bid from the agent, and the agent obtained a smaller load for a higher price. On the SAW market with online update, the agent was able to accurately update its bid to a near optimal schedule.

\section{DISCUSSION AND CONCLUSIONS}

The online information that is available to the intelligent agent clearly improves its position on the market, allowing it to obtain a higher utility. However, in adversarial settings other agents may use this information to speculate against this first agent. In the current experiment, no adversarial agents were considered, thus our insights transfer primarily to cooperative

\section{Case 1: without wind (w/o)}

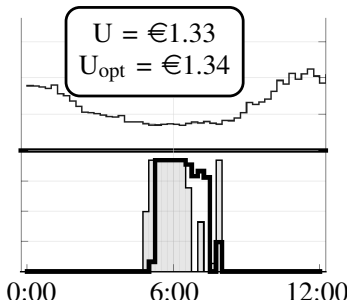

(a) CSW marke
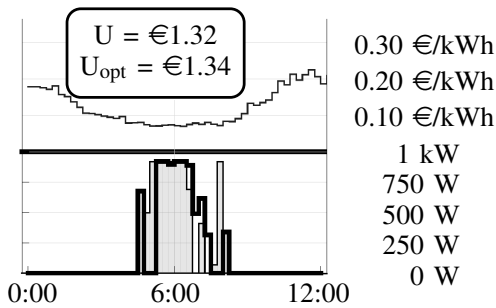

(b) SAW market (offline price forecasts only)
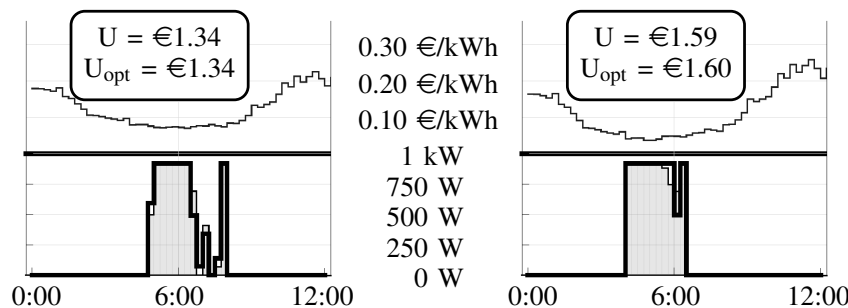

(c) SAW market (online price forecasts)

\section{Case 2: with wind (w)}
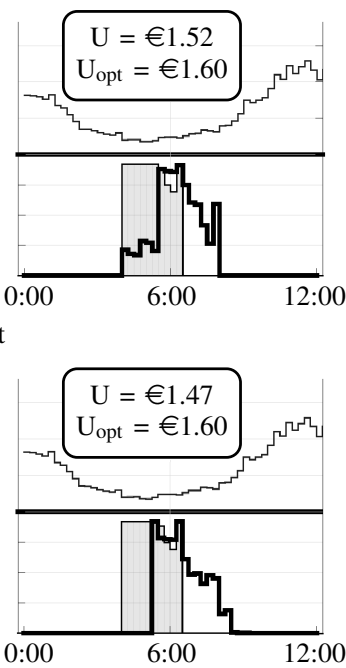

$12: 00$
. Claessen, B. Claessens, M.P.F. Hommelberg, A. Molderink, V. Bakker, H.A. Toersche and M.A. van den Broek, "Comparative analysis of tertiary control systems for smart grids using the Flex Street model," Renewable Energy, vol. 69, pp. 260-270, ISSN 0960-1481, Sep. 2014.

[7] P. Cramton and S. Stoft, "Why we need to stick with uniform-price auctions in electricity markets," The Electricity Journal, vol. 20, issue 1 , pp. 26-37, 2007.

[8] A. Ockenfels, V. Grimm and G. Zoettl, "Electricity market design," English translation by EEX AG, 2008.

[9] V. Robu, S. Stein, E.H. Gerding, D.C. Parkes, A. Rogers and N.R. Jennings, "An online mechanism for multi-speed electric vehicle charging," in Proc. of the 2nd Int. Conf. on Auctions, Market Mechanisms and their

Applications (AMMA), pp. 100-112, 2011. response. This improved alignment of volatile generation with (flexible) loads yields higher profits for the individual, and has the potential to alleviate the system imbalances.

\section{REFERENCES}

1] N. Höning and H. La Poutré, "An electricity market with fast bidding, planning and balancing in smart grids," Multiagent and Grid Systems, market integrity and transparency," Official Journal of the European Union, L326/1, 2011.

] E.H. Gerding, R.K. Dash, A. Byde and N.R. Jennings, "Optimal strategies for bidding agents participating in simultaneous Vickrey auctions with pp. 939-982, 2008.

4] P. Cramton, Y. Shoham and R. Steinberg, "Combinatorial auctions," MIT

Yuzo Fujishima, David McAdams and Yoav Shoham, "Speeding up ascending-bid auctions," in Proc. of the 16th Int. Joint Conf. on Artificial Intelligence (IJCAI), Stockholm, Sweden, pp. 554-559, 1999.
Figure 6: Selected results (last day clearing prices and EV demand) showing the improved alignment of the procured schedule (solid black line) with the optimum (grey area) when the bidder considers online price information. This illustrates the value of (online) information for intelligent demand response. settings, e.g. local markets, where adversarial behaviour has flexible loads and, correspondingly, the number of intelligent are price taking. Individual market power can become large, especially in smaller markets. As the influence of bids on prices becomes a significant factor in the negotiation process, to what extent market prices are influenced by their own bids. Future research may investigate the influence of price information on agent utility when the agent's market impact is high.

This article presents simulation experiments in alternative Results show the utility obtained by an intelligent agent and relate it to an upper bound deduced from perfect knowledge of future prices. Our case study simulations show that a near SAW market, and uses the available online information about all timeslots. The value of this online information was highest prices were hard to predict, i.e. in a scenario with volatile uncertainty over available energy and corresponding prices, Press, 2006. 